\title{
Uso de Simulador de Geração de Tráfego para a Melhoria de Desempenho de Sistemas Legados Críticos
}

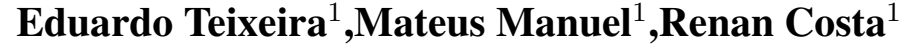 \\ ${ }^{1}$ Universidade de Brasília (UnB) - Brasília, DF - Brazil \\ \{eduhenr, mateusmanuel, renancf $\}$ dunb.br
}

\begin{abstract}
Some legacy systems tend to have a barely scalable architecture, making little progress on performance issues. On the other hand, access to the Internet has been increasing, making more people connected and therefore, forcing systems to maintain efficiency with the new demand. This work presents an architectural change carried out in the legacy registration system of the University of Brasília aiming at performance improvement. It is also shown a traffic generator (TRAFFICGEN), built to subject the system to a load similar that occurs during enrollment periods and thus various improvements were made. It has been found that the modified system has achieved a performance improvement of more than $5 \times$ compared to the production version with half the servers used and still supports a load increase of at least $215 \%$ without performance degradation.
\end{abstract}

Resumo. Alguns sistemas legados tendem a possuir uma arquitetura pouco escalável, fazendo com que pouco se possa evoluir em questões de desempenho. Por outro lado, o acesso a internet vem crescendo, fazendo com que mais pessoas tenham acesso e obrigando, consequentemente, que os sistemas evoluam para manter a eficiência com a nova demanda. Nesse trabalho é apresentada uma mudança arquitetural realizada no sistema legado de matrículas da Universidade de Brasília visando a melhoria de desempenho. Também é demonstrado um gerador de tráfego (TRAFFICGEN) construído para submeter o sistema a uma carga semelhante à que ocorre durante os períodos de matrícula e dessa forma fossem realizadas diversas melhorias. Foi verificado que o sistema modificado alcançou uma melhoria de desempenho de mais de $5 \times \mathrm{com}$ relação à versão de produção com a metade dos servidores utilizados e que ainda suporta um aumento de carga de pelo menos $215 \%$ sem degradação do desempenho.

\section{Introdução}

Grandes investimentos são realizados em sistemas de software e para que as organizações obtenham retorno desse investimento, o software deve ser utilizado por vários anos. O tempo de duração de sistemas de software é variável e pode permanecer por mais de 10 anos [Lehman 1980]. Muitos desses sistemas ainda são fundamentais para as organizações e qualquer falha desses serviços acarretará um sério efeito em seu dia a dia [Bennett and Rajlich 2000].

Ao longo dos últimos, em particular após o REUNI [Brasil 2007], a Universidade de Brasília (UnB) aumentou consideravelmente o quantitativo de cursos de graduação e, 
consequentemente, o número de alunos. Por outro lado, os sistemas apoio, como o MW, não acompanharam este ritmo de evolução e hoje apresentam deficiências no que tange ao desempenho durante a execução de atividades críticas como o processo de matrícula.

Neste processo, em uma das etapas finais, os alunos concorrem pelas vagas existentes obedecendo o critério First Come First Served[Tanenbaum and Wetherall 2010] (FCFS), tendo em vista haver um número maior de alunos do que vagas disponíveis em alguns cursos. Este critério, por sua vez faz com que haja um contingente de demanda ao servidor que supera 500 conexões concorrentes, o que por sua vez acarreta em problemas de indisponibilidade e lentidão no atendimento destas demandas. Este trabalho apresenta em detalhes as ações e técnicas utilizadas com o intuito de melhorar o desempenho deste sistema legado e permitir não apenas a sobrevida do sistema mas principalmente atender com maior celeridade as demandas em momentos de pico como o que ocorre durante o período de matrícula.

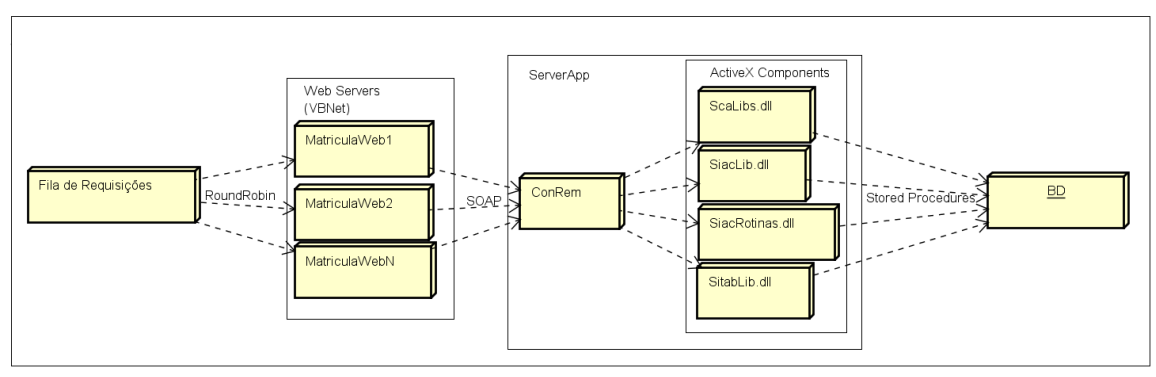

Figura 1. Arquitetura de Produção do MW.

Foi realizado o estudo da arquitetura de produção do MW, e foram identificados alguns pontos críticos. Um deles está relacionado ao tratamento da fila de requisições, que após o round robin entre os servidores Web (WebServers), é realizado por meio de um único servidor, o ServerApp, conforme mostrado na Figura 1. O ServerApp é um servidor de aplicação que contém o WebService ConnectionRemote (ConRem) que se comunica com os WebServers. O ConRem possui diversos componentes ActiveX que realizam o acesso ao banco de dados por meio de stored procedures que contém as regras de negócio da aplicação. Dessa forma, para que as operações sejam realizadas no servidor de banco de dados é necessário enviar as requisições ao ConRem por meio do protocolo SOAP, que por sua vez acessa os componente ActiveX que finalmente realizam chamadas para as stored procedures. Ou seja, existem limitações na escalabilidade da aplicação devido ao processamento de todas as requisições serem realizadas por um único servidor e também devido ao alto grau de overhead para que a aplicação persista os dados em banco. Além disso, caso ocorra uma queda ou um alto volume de requisições no ServerApp ocorre o comprometimento da qualidade de serviço e até mesmo do funcionamento para os usuários que estejam utilizando o sistema.

Um outro ponto crítico encontrado nessa arquitetura se deve ao fato de que todas as regras de negócio do sistema se encontram em stored procedures escritas na linguagem Transact-SQL (T-SQL). Essas stored procedures são chamadas em cadeia e utilizam tabelas temporárias para a comunicação entre elas. $\mathrm{O}$ acesso concorrente de muitas chamadas a stored procedures gera um alto consumo de CPU no ServerApp e consequentemente lentidão no processamento da fila de requisições. Além disso, o uso de stored proce- 
dures para regras de negócio dificulta o entendimento e a depuração do código, pois as ferramentas de debugging para a linguagem T-SQL são muito limitadas, podendo produzir resultados diferentes dos ambientes próprios de linguagens de programação. Devido a esse modo de funcionamento, é frequente o estouro de vagas em ofertas de disciplinas sendo necessária a intervenção manual dos coordenadores dos departamentos para realizar os ajustes necessários para cada disciplina ofertada.

\section{Métodos}

Foi proposta uma arquitetura alternativa ao modelo atual mostrado na Figura 1. Nessa arquitetura foi retirada da aplicação a comunicação com o WebService ConRem, por ser identificado arquiteturalmente como um ponto único de gargalo e de falha. Por meio dessa retirada existe uma maior abertura para o paralelismo nas execuções das consultas ao banco de dados que podem ser realizadas diretamente pela aplicação. Além disso, caso ocorra a falha de um WebServer não ocorre o comprometimento de todo o sistema, afetando apenas os usuários conectados no IIS em que ocorreu a queda [Coulouris et al. 2011].

Além da retirada do ConRem foi realizada a conversão do projeto e de suas dependências para o compilador Visual Studio 2015 com o objetivo de melhorar o desempenho do binário executável gerado e da sua utilização com a versão mais recente do IIS (Internet Information Server 8.5). O IIS 8.5 realiza o gerenciamento de múltiplas conexões simultâneas de forma mais otimizada e pode ser utilizado com um sistema operacional mais recente, como o Windows $2012 \mathrm{R} 2$, que também possui um melhor desempenho para gerenciamento de recursos de I/O e CPU.

Também foi realizada a conversão do código de T-SQL para VBNet por ser a mesma linguagem utilizada no site e com o objetivo de melhorar o entendimento das regras de negócio e da depuração do código, estudar potenciais pontos de melhoria e efetuar manutenções evolutivas e corretivas mais rapidamente. Entretanto, o uso de tabelas temporárias foi mantido pois a sua conversão implicaria em um alto risco devido a grande quantidade de regras de negócio envolvidas.

Foi realizada a modificação de todas as consultas em banco (select) utilzando a cláusula with(nolock) para diminuição das serializações em função da grande quantidade de consultas realizadas pelo processo de matrícula. Também foi utilizada a estratégia de locks de linha with(updlock) transacional para atualização das tabelas de oferta e reserva de vagas em disciplinas para evitar o estouro de vagas.

\section{Resultados}

A arquitetura proposta foi implantada em produção no segundo período de 2017 (2017/2). Foram utilizados 12 WebServers com Windows Server 2012 R2, 16 gigabytes de memória e 16 núcleos cada. Devido a conversão de código existente de T-SQL para VBNet ocorreram 2 bugs no período de matrícula, por conta do comportamento específico da linguagem T-SQL ao tratar variáveis não inicializadas. Entretanto, esses problemas foram corrigidos rapidamente e as atualizações em ambiente de produção foram efetuadas no mesmo dia da requisição, o que comprovou o alcance da melhoria almejada com relação ao melhor entendimento e correções rápidas. Além disso, também verificou-se que não ocorreram mais os estouros de vagas, pois a crítica da matrícula para a aquisição de vagas é realizada 
atomicamente. Para a Arquitetura Proposta foram obtidos os resultados da Figura 2(a). Apesar da melhoria de desempenho do sistema com a "Arquitetura Proposta"ser $2.59 \times$ superior nas primeiras 2 horas, ocorreram problemas de timeout de banco, o que gerou lentidão e erros de acesso na primeira hora. Isso foi constatado pelo comportamento linear da "Arquitetura Proposta"com relação à quantidade de matrículas efetuadas no tempo e ocorreu devido ao grande volume de requisições concorrentes dos estudantes pelas vagas remanescentes.

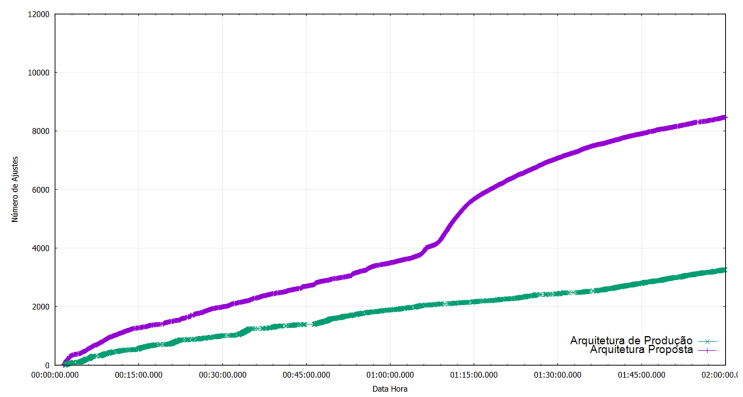

(a) Desempenho das Arquiteturas de Produção e Proposta.

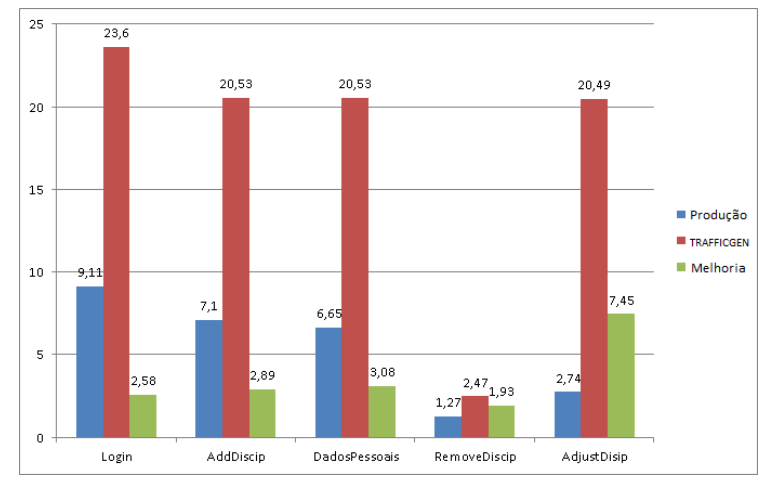

(c) Resultados Obtidos com os Testes de Stress

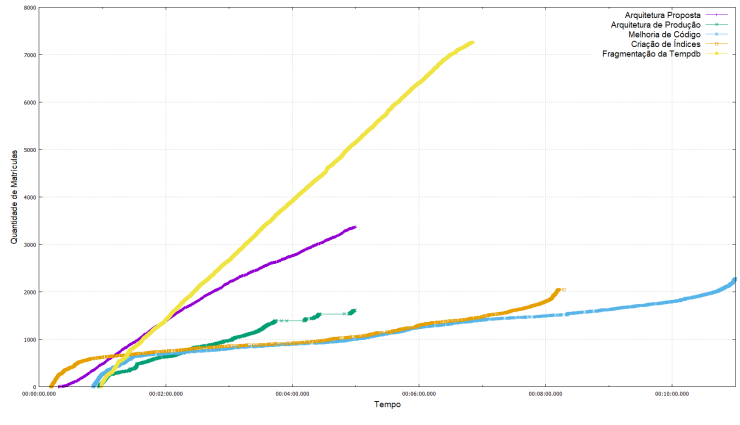

(b) Resultados Obtidos com os Testes de Stress.

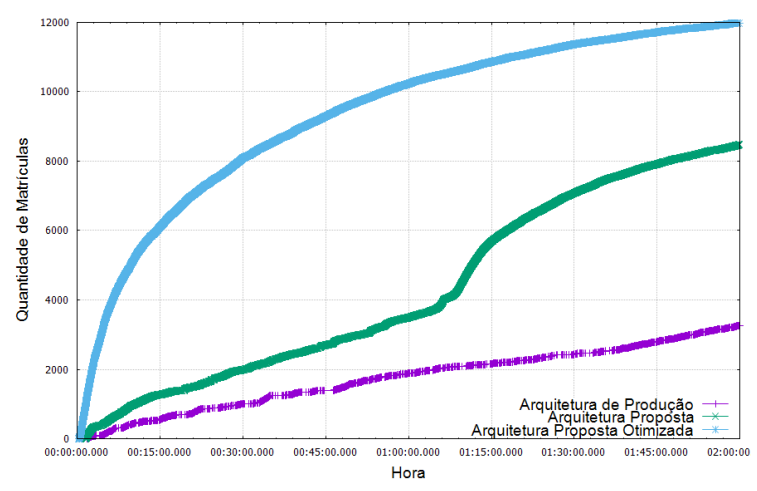

(d) Desempenho das Arquiteturas de Produção e Proposta e Otimizada.

Figura 2. Comportamento da Quantidade de Matrículas no Tempo.

Devido a esse problema de desempenho que ocorreu na primeira hora de funcionamento da Arquitetura Proposta foi desenvolvido um simulador escrito na linguagem C++ para gerar tráfego com um volume de requisições parecido com o que ocorre durante o sistema em produção. Esse gerador de tráfego, denominado TRAFFICGEN, realiza a carga para a memória de todas as matrículas do período 2017/2 e realiza os POSTs HTTP utilizando várias conexões TCP concorrentes originadas de várias threads e processos que se comunicam por meio de Message Passing Interface (MPI) [Cameron Hughes 2003]. Os buffers dos POSTs foram capturados com o sniffer Wireshark durante a operação das funcionalidades consideradas mais importantes: login; obtenção de dados pessoais; remoção de disciplinas; adição de disciplinas, sendo essa a mais crítica. Dessa forma, esses buffers puderam ser enviados mudando apenas as informações relativas ao número de matrícula, disciplina e turma de cada aluno. Com o uso do TRAFFICGEN foram realizados os testes de eficiência e de stress.

Com os testes de stress submetidos por meio do TRAFFICGEN foi possível en- 
contrar diversos problemas de desempenho a cada bateria de testes e realizar vários ajustes para resolvê-los, como é possível observar na Figura 2(b). O envio de requisições do TRAFFICGEN para os testes de stress foi realizado em rajada durante 5 minutos, sendo encerrada a geração de tráfego após esse intervalo, porém esperando o resultado das requisições enfileiradas até o término de todas as threads e processos iniciados, demorando cerca de 11 minutos para a execução no pior caso, como pode ser visto na Figura 2(b). Dessa forma, na Figura 2(b) o eixo $x$ corresponde ao tempo em minutos da geração de tráfego e espera pelo término das threads e processos iniciados pelo TRAFFICGEN, enquanto que o eixo y corresponde ao número de matrículas realizadas. Foi utilizado o intervalo de 5 minutos pois este tempo foi suficiente para verificar o comportamento do tempo médio e executar várias baterias de testes por ser um tempo curto. Entre as melhorias efetuadas as que tiveram os melhores resultados foram a melhoria do código-fonte (em azul), a criação de novos índices em tabelas (em laranja) e a fragmentação do banco de dados responsável pelo tratamento de tabelas temporárias (em amarelo). Na Figura 2(b) também é possível observar e comparar os resultados das otimizações realizadas com o comportamento das Arquiteturas de Produção (em verde) e Proposta (em roxo) durante o funcionamento durante os primeiros 5 minutos que ocorreram na Figura 2(a). Entre as melhorias de código-fonte foram realizadas o refactoring da rotina de login, a retirada do uso de views e a reescrita das consultas especificando as colunas necessárias ao invés de todas com o uso de "*", sendo esta última um uso recomendado pelas melhores práticas no que tange ao SQL. Além disso, foi modificado o acesso aos cursores dos resultsets para o modo sem serialização e sem cópia. Cada uma dessas melhorias foi realizada cumulativamente na ordem: melhoria do código-fonte, criação de novos índices e a fragmentação das tabelas temporárias.

Como pode ser visto, o maior ganho obtido ocorreu com o uso de fragmentação das tabelas temporárias, pois apesar da simplificação arquitetural efetuada, o sistema modificado ainda utiliza intensamente tabelas temporárias para a realização das regras de negócio. Durante o tempo em que o teste de stress do TRAFFICGEN gerou carga foram realizadas 7.264 matrículas no melhor caso para a modificação "Fragmentação da TempDb"(em amarelo na Figura 2(b)), enquanto que na "Arquitetura Proposta"foram realizadas 3.368 matrículas (em roxo na Figura 2(b)), sendo este último obtido dos primeiros 5 minutos de funcionamento real em ambiente de produção obtido da Figura 2(a). Dessa forma, o sistema ainda suporta um aumento de carga de pelo menos $215 \%$, sem degradação do desempenho. O aumento de carga suportado pode até ser maior que os $215 \%$, pois além de o ambiente de produção possuir um poder computacional maior do que o do TRAFFICGEN, a carga real a qual o sistema foi submetido foi inferior à do ambiente do gerador de tráfego, sendo este submetido a 1440 conexões simultâneas, enquanto que o sistema real alcançou o pico de 520 conexões simultâneas.

No ambiente do TRAFFICGEN esses ajustes alcançaram uma melhoria $7.45 \times$ superior para a funcionalidade mais crítica (adição de disciplina) e em média $2.6 \times$ superior para as demais funcionalidades, como pode ser visto na Figura 2(c), onde as barras vermelhas e azuis correspondem ao número de operações por segundo (eixo $y$ ) para cada funcionalidade (eixo $x$ ) e a barra verde corresponde ao ganho obtido. Dessa forma, para a realização das matrículas no primeiro semestre letivo de 2018 (2018/1) foi realizada a diminuição dos WebServers de produção pela metade, por ser essa a menor média de ganho encontrada, como mostrado na Figura Figura 2(c). 
As otimizações realizadas com o apoio do gerador de tráfego TRAFFICGEN foram aplicadas na "Arquitetura Proposta"e foi obtido o resultado da "Arquitetura Proposta Otimizada", conforme mostrado na Figura 2(d), onde o eixo $x$ corresponde à hora do dia a partir da liberação de funcionamento do sistema que ocorreu às 00:00hs e o eixo $y$ corresponde ao número de matrículas efetuadas.

É possível observar que o sistema após as alterações efetuadas teve um comportamento logarítmico ao invés de linear com relação ao número de matrículas no tempo, levando a uma melhoria de desempenho $2.92 \times$ superior na primeira hora se comparado com a "Arquitetura Proposta", e 5.41× em relação à "Arquitetura de Produção".

\section{Conclusão}

A melhoria de desempenho em sistemas distribuídos nem sempre está associada ao aumento da capacidade de processamento do hardware, sendo que os maiores ganhos geralmente ocorrem em mudanças no modo de funcionamento da arquitetura desses sistemas [Tanenbaum and Steen 2008].

Este trabalho mostrou ser possível, com o auxílio de um simulador de geração de tráfego, simplificar a arquitetura e diminuir pela metade o número de servidores do sistema legado MW e mesmo assim obter um ganho de desempenho $5.41 \times$ maior do que a antiga versão. Além disso, o sistema ainda suporta um aumento de pelo menos $215 \%$ na carga sem comprometer a qualidade no desempenho.

Dessa forma, foi possível dar uma sobrevida a um sistema legado crítico sem necessariamente reescrever toda a aplicação, o que teria um custo muito maior do que a escrita do TRAFFICGEN. Esses resultados alcançados geraram uma grande satisfação da comunidade acadêmica com o Sistema de Matrículas da Universidade de Brasília no primeiro período letivo de 2018.

\section{Referências}

Bennett, K. H. and Rajlich, V. T. (2000). Software maintenance and evolution: a roadmap. In Proceedings of the Conference on the Future of Software Engineering, pages 73-87. ACM.

Brasil (2007). Decreto $\mathrm{n}^{0} 6.096$ de 24 de abril de 2007a. http: / /www . planalto. gov.br/ccivil_03/_ato2007-2010/2007/decreto/d6096.htm. Accessed: 2017-03-28.

Cameron Hughes, T. H. (2003). Parallel and Distributed Programming Using C++. Editora Addison Wesley, first edition.

Coulouris, G., Dollimore, J., Kindberg, T., and Blair, G. (2011). Distributed Systems: Concepts and Design. Addison-Wesley Publishing Company, USA, 5th edition.

Lehman, M. M. (1980). Programs, life cycles, and laws of software evolution. Proceedings of the IEEE, 68(9):1060-1076.

Tanenbaum, A. S. and Steen, M. V. (2008). Distributed systems: principles and paradigms. Editora Pearson Prentice Hall, second edition.

Tanenbaum, A. S. and Wetherall, D. J. (2010). Computer Networks. Editora Pearson Prentice Hall, fifth edition. 